\title{
Quasi-Random Hypergraphs Revisited
}

\author{
Fan Chung* \\ University of California, San Diego, California; e-mail: fan@ucsd.edu
}

Received 20 April 2010; accepted 16 March 2011; received in final form 17 March 2011

Published online 8 November 2011 in Wiley Online Library (wileyonlinelibrary.com).

DOI 10.1002/rsa.20388

\begin{abstract}
The quasi-random theory for graphs mainly focuses on a large equivalent class of graph properties each of which can be used as a certificate for randomness. For $k$-graphs (i.e., $k$ uniform hypergraphs), an analogous quasi-random class contains various equivalent graph properties including the $k$-discrepancy property (bounding the number of edges in the generalized induced subgraph determined by any given $(k-1)$-graph on the same vertex set) as well as the $k$-deviation property (bounding the occurrences of "octahedron", a generalization of 4-cycle). In a 1990 paper (Chung, Random Struct Algorithms 1 (1990) 363-382), a weaker notion of $l$-discrepancy properties for $k$-graphs was introduced for forming a nested chain of quasi-random classes, but the proof for showing the equivalence of $l$-discrepancy and $l$-deviation, for $2 \leq l<k$, contains an error. An additional parameter is needed in the definition of discrepancy, because of the rich and complex structure in hypergraphs. In this note, we introduce the notion of $(l, s)$-discrepancy for $k$-graphs and prove that the equivalence of the $(k, s)$-discrepancy and the $s$-deviation for $1 \leq s \leq k$. We remark that this refined notion of discrepancy seems to point to a lattice structure in relating various quasi-random classes for hypergraphs. (C) 2011 Wiley Periodicals, Inc. Random Struct. Alg., 40, 39-48, 2012
\end{abstract}

Keywords: quasi-random; discrepancy; deviation

\section{INTRODUCTION}

The study of quasi-random graphs and hypergraphs explores the relationship among properties of graphs with special emphasis of finding equivalence classes and their classifications. For graphs, there is a large equivalence class that includes the discrepancy property and the deviation property [4]. The discrepancy property for a graph $G$ is associated with bounding the difference between the number of edges in an induced subgraph $S$ of $G$ and the expected number of edges in $S$ (which is basically $|S|^{2} / 4$ for a graph $G$ with edge density $1 / 2$ ). The discrepancy property for $G$ is associated with bounding the difference between the number

Correspondence to: F. Chung

Corrigendum: "Quasi-random classes of hypergraphs," Random Structures and Algorithms 1 (1990), 363-382.

*Supported by ONR MURI (N000140810747), AFSOR 9550-09-1-0900.

(C) 2011 Wiley Periodicals, Inc. 
of four cycles containing an even number of edges in $G$ and those with an odd number of edges in $G$. To extend the study of quasi-random graphs to $k$-uniform hypergraphs, (or $k$-graphs for short), there have been numerous attempts $[1,3,5,7,8]$. In the effort to extend the notion of deviation to $k$-graphs for $k \geq 3$, there is a nested sequences of $l$-deviation $\operatorname{dev}_{l}$, $2 \leq l \leq k$ which concern the counts of so-called even "octahedra" and odd octahedra on $2 l$ vertices. To generalize the notion of discrepancy for a $k$-graphs $H$ with vertex set $V$, one of the ways is to consider the $l$-discrepancy $\operatorname{disc}_{l} H$, for a fixed $l, 2 \leq l \leq k$, which concerns the maximum difference of the edge counts in subgraph of $H$ induced by any $(l-1)$-graph $G$ from the expected value over all $G$ on $V$. In $[1,3]$ it was shown that for a $k$-graph $H$, the property disc $H=\operatorname{disc}_{k} H$ and $\operatorname{dev} H=\operatorname{dev}_{k} H$ are equivalent in the sense that for any $\epsilon$ there exists $\delta$ such that $\operatorname{disc} H \leq \delta$ implies $\operatorname{dev} H \leq \epsilon$, (denoted by disc $\Rightarrow$ dev) and the reverse direction holds as well.

To further understand the structure for $k$-graphs, a natural approach is to establish a nested sequence of equivalence classes. In [1], it was shown that for $2 \leq l \leq k, \operatorname{dev}_{l} \Rightarrow \operatorname{disc}_{l}$. However, the proof for $\operatorname{disc}_{l} \Rightarrow \operatorname{dev}_{l}$ contains two cases, one of which, namely for $2 \leq l<k$, contains an erroneous statement. A counterexample was given in [6]. As it turns out, the hypergraphs have a richer and more intriguing structure than previously suspected (by the author). There are further extensions of the discrepancy property which we call $(l, s)$ discrepancy, denoted by $\operatorname{disc}_{l}^{(s)} H$, for a $k$-graph $H$ with vertex set $V$, where $2 \leq l \leq k$ and $1 \leq s \leq\left(\begin{array}{l}k \\ l\end{array}\right)$. Roughly speaking, $\operatorname{disc}_{l}^{(s)} H$ concerns the subgraphs $S_{s}$ of $H$ which are induced by an $l$-graph $G$ on $V$ in the sense that an edge $x$ in $E(H)$ is in $S_{s}$ if the number of $l$-edges in $G$ contained in $x$ is at least $s$. The previous notion of $\operatorname{disc}_{l}$ is the special case of disc ${ }_{l}^{(s)}$ with $s=\left(\begin{array}{c}k \\ l-1\end{array}\right)$. The paper [6] examines the case of $\operatorname{disc}_{2}^{(k)}$ which was then shown to belong to a large equivalence class of hypergraph properties including counting the appearances of a fixed "linear" $k$-graph $F$ in $H$ where "linear" means the restriction that any two edges in $F$ intersect at most one vertex.

With this refined notion of $(l, s)$-discrepancy for $k$-graphs, numerous questions arise. How are various known hypergraph properties related to $\operatorname{disc}_{l}^{(s)}$ ? For example, suppose we consider a generalization of linear $k$-graphs. We say a $k$-graph $F$ is $l$-linear if any two edges in $F$ intersect at no more than $l$ vertices. We can then define the following subgraph containment property for a $k$-graph $H$ on $n$ vertices :

$\mathrm{P}_{l}$ : For every $(l-1)$-linear $k$-graph $F$ on $r$ vertices with $r$ vertices and $t$ edges with $r \geq k$, the number $N_{F}(H)$ of labelled embeddings of $F$ in $H$ satisfies

$$
N_{F}(H)=(1 / 2)^{t} n^{r}+o\left(n^{r}\right)
$$

It seems plausible to conjecture that $P_{l}$ is equivalent to $\operatorname{disc}_{l}^{(s)}$ with $s=\left(\begin{array}{c}k \\ l-1\end{array}\right)$ by extending the techniques in [6] for the case of $l=2$. Although the above formulation is mainly for $k$-graph $H$ with edge density $1 / 2$, a general definition for $\mathrm{P}_{l}$ with graphs with edge density $p$ can be obtained in a straightforward manner by replacing $1 / 2$ by $p$.

There are further questions just for the case of $l=2$. Even for the special case of $k=3$ and $s=2$, the discrepancy property for a 3-graph $H$ is reduced to the following: For any subset $S$ of vertices, the number of edges in $H$ containing at least 2 vertices in $S$ is about as expected. Will this property be equivalent to some modified version of the deviation property (similar to some partial "doubling" as described in [6])?

In general, for various given hypergraph properties, can they be related to the $\operatorname{disc}_{l}^{(s)}$ in some way? Do they form quasi-random equivalence classes? What are the hierarchy of these quasi-random classes? And, how effective are these properties to be used as certificates for 
randomness? To partially answer some of these questions, we show that $\operatorname{dev}_{s}$ is equivalent to $\operatorname{disc}_{k}^{(s)}$ for $k$-graphs in the remaining part of this note. Further questions and remarks concerning the lattice structure of quasi-random classes for $k$-graphs will be discussed in the last section.

\section{A REFINED NOTION OF THE DISCREPANCY PROPERTIES FOR HYPERGRAPHS}

We follow the notation in [3]. A $k$-uniform hypergraph $H=\left(V, \mu_{H}\right)$ consists of a set of $V$ of vertices of $H$ together with a function $\mu_{H}:\left(\begin{array}{l}V \\ k\end{array}\right) \rightarrow\{1,-1\}$, called the multiplicative edge function of $H$. The set $E(H)=\mu_{H}^{-1}(-1)$ is called the edge set of $H$. When there is no confusion, we call $H$ a $k$-graph. For a given function $\mu:\left(\begin{array}{l}V \\ k\end{array}\right) \rightarrow\{1,-1\}$, denote by $\bar{\mu}$ the extension $\bar{\mu}: V^{k} \rightarrow\{1,-1\}$ by $\bar{\mu}\left(v_{1}, \ldots, v_{k}\right)=\mu\left(\left\{v_{1}, \ldots, v_{k}\right\}\right)$ where $v_{1} \ldots, v_{k}$ are distinct elements of $V$ and 1 otherwise.

Definition. The l-deviation of a $k$-graph $H=H(E, V)$ with $|V|=n$, denoted by $\operatorname{dev}_{l} H$, is defined by

$$
\operatorname{dev}_{l} H=\frac{1}{n^{k+l}} \sum_{\substack{v_{i}\left(0, v_{i}(1) \in V \\ 1 \leq i \leq l\right.}} \sum_{\substack{w_{j} \in V \\ l+1 \leq j \leq k}} \prod_{\substack{\epsilon_{i} \in\{0,1\} \\ 1 \leq i \leq l}} \bar{\mu}_{H}\left(v_{1}\left(\epsilon_{1}\right), \ldots, v_{l}\left(\epsilon_{l}\right), w_{l+1}, \ldots, w_{k}\right)
$$

where $\bar{\mu}(x)=-1$ if $x$ is an edge in $H$ and $\bar{\mu}(x)=1$ otherwise.

We remark that the above definition can be generalized to focus on graphs with edge density $p$ by defining $\mu(x)=-p$ if $x$ is an edge in $H$ and $\mu(x)=1-p$ otherwise.

Definition. For a $k$-graph $H$ and a l-graph $G$ on the same vertex set $V$, we define

$$
\begin{aligned}
& E(H, G)=\left\{x \in E(H):\left(\begin{array}{l}
x \\
l
\end{array}\right) \subseteq E(G)\right\}, \\
& e(H, G)=k !|E(H, G)| .
\end{aligned}
$$

Namely e $(H, G)$ counts the number of ordered subsets in $E(H, G)$.

Definition. For a $k$-graph $H$ on vertex set $V$ with $|V|=n$, we define $\operatorname{disc}_{l} H$ as follows:

$$
\operatorname{disc}_{l} H=\frac{1}{n^{k}} \max _{G}|e(H, G)-e(\bar{H}, G)|,
$$

where the maximum is taken over all $(l-1)$-graphs $G$ on $V$.

It was shown in $[1,3]$ that

$$
\operatorname{dev}_{l} H \geq\left(\operatorname{disc}_{l} H\right)^{2^{l}}
$$

and for $l=k$,

$$
\operatorname{dev}_{k} H \leq 4^{k}\left(\operatorname{disc}_{k} H\right)^{2^{-k}}
$$


For a $k$-graph $H$, we use the notation that $\operatorname{dev} H=\operatorname{dev}_{k} H$ and $\operatorname{disc} H=\operatorname{disc}_{k}^{(k)} H$ which [3] mainly focused on.

It would have led to quasi-random classes for hypergraphs if a similar statement as follows holds for $2 \leq l<k$.

$$
\operatorname{dev}_{l} H \leq 4^{l}\left(\operatorname{disc}_{l} H\right)^{2^{-l}} .
$$

However, this inequality is not true for $l \neq k$ as evidenced by the example given int [6]. So, a natural question is to find the 'right' equivalent discrepancy property for $\operatorname{dev}_{l}$.

Definition. For a k-graph $H$ and an l-graph $G$, we define

$$
\begin{aligned}
& E_{s}(H, G)=\left\{x \in E(H):\left|\left(\begin{array}{l}
x \\
l
\end{array}\right) \cap E(G)\right| \geq s\right\}, \\
& e_{s}(H, G)=k !\left|E_{s}(H, G)\right| .
\end{aligned}
$$

Namely $e_{s}(H, G)$ counts the number of ordered subsets in $E_{l}(H, G)$. We note that for the case of $l=k-1$ and $s=k$, we have $e(H, G)=e_{k}(H, G)$.

Definition. For a $k$-graph $H$ on $n$ vertices, we define $\operatorname{disc}_{l}^{(s)} H$ as follows:

$$
\operatorname{disc}_{l}^{(s)} H=\frac{1}{n^{k}} \max _{G}\left|e_{s}(H, G)-e_{s}(\bar{H}, G)\right|,
$$

where the max is taken over all $(l-1)$-graphs $G$ on $V$.

Note that disc $H$ is the special case $\operatorname{disc}_{k}=\operatorname{disc}_{k}^{(k)}$ and disc $\operatorname{dis}_{l}$ the special case $\operatorname{disc}_{l}=$ $\operatorname{disc}_{l}^{(s)}$ for $s=\left(\begin{array}{c}k \\ l-1\end{array}\right)$.

We remark that the above definition can be modified to focus on graphs with density $p$ by defining $\operatorname{disc}_{l}^{(s)} H=\frac{1}{\operatorname{vol} H} \max _{G}\left|e_{s}(H, G)-p \cdot e_{s}\left(\left(\begin{array}{l}V \\ k\end{array}\right), G\right)\right|$ where $G$ ranges over all $(l-1)$ graphs. Here vol $H$ denotes the number of edges in $H$. For simplicity, we will mainly deal with the case of $p=1 / 2$ here.

Although we are far from fully understanding the relationship among properties $\operatorname{disc}_{l}^{(s)}$, certain implications can be derived for the case of $l=k$. To simplify the notation, we write

$$
\operatorname{disc}^{(s)} H=\operatorname{disc}_{k}^{(s)} H .
$$

Note that for $\operatorname{disc}^{(s)}$, the interesting range for $s$ is for $s \leq k$.

We will prove the following two theorems to establish the equivalence implications of $\operatorname{dev}_{s}$ and $\operatorname{disc}^{(s)}$.

\section{THE $s$-DEVIATION PROPERTY IMPLIES THE DISCREPANCY PROPERTY $\operatorname{disc}^{(s)}$}

Theorem 1. For a $k$-graph $H$ and $2 \leq s \leq k$, we have

$$
\operatorname{dev}_{s} H \geq\left(\operatorname{disc}^{(s)} H\right)^{2^{s}} .
$$

Proof. It suffices to show that for any given $(k-1)$-graph $G$, we have

$$
\operatorname{dev}_{s} H \geq\left(E_{s}(H, G)-E_{s}(\bar{H}, G)\right)^{2^{s}} .
$$


This can be proved by applying the Cauchy-Schwarz inequality on selected terms repeatedly as follows. We consider

$$
\begin{aligned}
\operatorname{dev}_{s} H & =\frac{1}{n^{k+s}} \sum_{\substack{v_{i}(0), v_{i}(1) \in V \\
1 \leq i \leq s}} \sum_{\substack{w_{j} \in V \\
s+1 \leq j \leq k}} \prod_{\substack{\epsilon_{i} \in\{0,1\} \\
1 \leq i \leq s}} \bar{\mu}_{H}\left(v_{1}\left(\epsilon_{1}\right), \ldots, v_{s}\left(\epsilon_{s}\right), w_{s+1}, \ldots, w_{k}\right) \\
& =\frac{1}{n^{k+s}} \sum_{\substack{v_{i}(0), v_{i}(1) \in V \\
1 \leq i \leq s-1}} \sum_{\substack{w_{j} \in V \\
s+1 \leq j \leq k}}\left(\sum_{\substack{v \in V \\
\epsilon_{i} \in\{0,1\} \\
1 \leq i \leq s-1}} \bar{\mu}_{H}\left(v_{1}\left(\epsilon_{1}\right), \ldots, v_{s-1}\left(\epsilon_{s-1}\right), v, w_{s+1}, \ldots, w_{k}\right)\right)^{2} \\
& \geq \frac{1}{n^{k+s}} \sum_{\substack{w_{j} \in V \\
s+1 \leq j \leq k}} \sum_{\substack{v_{i}(0), v_{i}(1) \in V \\
1 \leq i \leq s-1}}^{G, s}\left(\sum_{v \in V} \prod_{\substack{\epsilon_{i} \in\{0,1\} \\
1 \leq i \leq s-1}} \bar{\mu}_{H}\left(v_{1}\left(\epsilon_{1}\right), \ldots, v_{s-1}\left(\epsilon_{s-1}\right), v, w_{s+1}, \ldots, w_{k}\right)\right)^{2}
\end{aligned}
$$

where $\sum^{G, s}$ denotes a partial sum with the restriction that the $v_{i}\left(\epsilon_{i}\right)$ satisfy the property that $\left(v_{1}\left(\epsilon_{1}\right), \ldots, v_{s-1}\left(\epsilon_{s-1}\right), w_{s+1}, \ldots, w_{k}\right)$ are edges in $G$ for all $\epsilon_{i}$. Thus we have

$$
\begin{aligned}
\operatorname{dev}_{s} H & \geq \frac{1}{n^{k+s-2}} \sum_{\substack{w_{j} \in V \\
s+1 \leq j \leq k}} \sum_{\substack{v_{i}(0), v_{i}(1) \in V \\
1 \leq i \leq s-1,}}^{G, s}\left(\frac{1}{n} \sum_{\substack{v \in V \\
v \in V}} \prod_{\substack{\epsilon_{i} \in\{0,1\} \\
1 \leq i \leq s-1}} \bar{\mu}_{H}\left(v_{1}\left(\epsilon_{1}\right), \ldots, v_{s-1}\left(\epsilon_{s-1}\right), v, w_{s+1}, \ldots, w_{k}\right)\right)^{2} \\
& \geq\left(\frac{1}{n^{k+s-1}} \sum_{\substack{w_{j} \in V \\
s \leq j \leq k}} \sum_{\substack{v_{i}(0), v_{i}(1) \in V \\
1 \leq i \leq s-1,1}}^{G, s} \prod_{\substack{\epsilon_{i} \in\{0,1\} \\
1 \leq i \leq s-1}} \bar{\mu}_{H}\left(v_{1}\left(\epsilon_{1}\right), \ldots, v_{s-1}\left(\epsilon_{s-1}\right), w_{s}, w_{s+1}, \ldots, w_{k}\right)\right)^{2} .
\end{aligned}
$$

We will repeat the same methods using the notation that $\sum^{G,[j, s]}$ denotes a partial sum with the restriction that the $v_{i}\left(\epsilon_{i}\right)$ satisfy the property that $\left(v_{1}\left(\epsilon_{1}\right), \ldots, v_{j-1}\left(\epsilon_{j-1}\right), w_{j}, \ldots, w_{t-1}, w_{t+1}, \ldots, w_{k}\right)$ are edges in $G$ for all $\epsilon_{i}$ and $t \in[j, s]$. Then we have

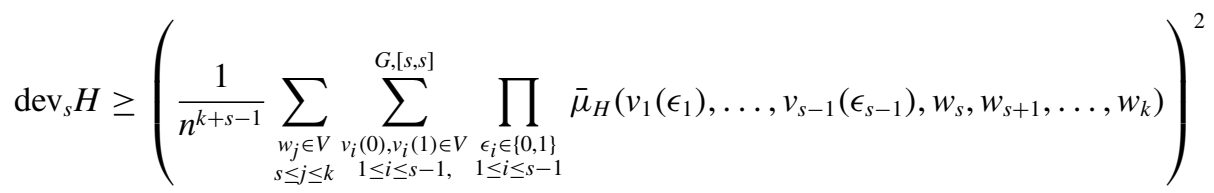

$$
\begin{aligned}
& \geq\left(\frac{1}{n^{k+s-2}} \sum_{\substack{w_{j} \in V \\
s-1 \leq j \leq k}} \sum_{\substack{v_{i}(0), v_{i}(1) \in V \\
1 \leq i \leq s-2,}}^{G,[s-1, s]} \prod_{\substack{\epsilon_{i} \in\{0,1\} \\
1 \leq i \leq s-2}} \bar{\mu}_{H}\left(v_{1}\left(\epsilon_{1}\right), \ldots, v_{s-1}\left(\epsilon_{s-2}\right), w_{s}, w_{s+1}, \ldots, w_{k}\right)\right)^{4} \\
& \geq \ldots
\end{aligned}
$$




$$
\geq\left(\frac{1}{n^{k+1}} \sum_{\substack{w_{j} \in V \\ 2 \leq j \leq k}} \sum_{v_{1}(0), v_{1}(1) \in V}^{G,[2, s]} \prod_{\epsilon_{1} \in\{0,1\}} \bar{\mu}_{H}\left(v_{1}\left(\epsilon_{1}\right), w_{2}, w_{s+1}, \ldots, w_{k}\right)\right)^{2^{s-1}} .
$$

Therefore we have

$$
\begin{aligned}
\operatorname{dev}_{s} H & \geq\left(\frac{1}{n^{k-1}} \sum_{\substack{w_{j} \in V \\
2 \leq j \leq k}}\left(\frac{1}{n} \sum_{v \in V}^{G,[2, s]} \bar{\mu}_{H}\left(v, w_{2}, w_{s+1}, \ldots, w_{k}\right)\right)^{2}\right)^{2^{s-1}} \\
& \geq\left(\frac{1}{n^{k}} \sum_{\substack{w_{j} \in V \\
1 \leq j \leq k}}^{G,[1, s]} \bar{\mu}_{H}\left(w_{1}, w_{2}, w_{s+1}, \ldots, w_{k}\right)\right)^{2^{s}} \\
& =\left(\frac{1}{n^{k}}\left(e_{s}(H, G)-e_{s}(\bar{H}, G)\right)^{2^{s}}\right.
\end{aligned}
$$

for any $(k-1)$-graph $G$. Thus we conclude that

$$
\operatorname{dev}_{s} H \geq\left(\operatorname{disc}^{(s)} H\right)^{2^{s}} .
$$

\section{THE DISCREPANCY PROPERTY disc $^{(s)}$ IMPLIES THE $s$-DEVIATION PROPERTY}

Theorem 2. For a $k$-graph $H$ and $2 \leq s \leq k$, suppose that for every $(k-1)$-graph $G$ on $V$,

$$
\left|e_{s}(H, G)-e_{s}(\bar{H}, G)\right| \leq \epsilon n^{k} .
$$

Then we have

$$
\operatorname{dev}_{s} H \leq 2^{k+s} \epsilon^{1 /\left((k-s)(k-s+1) 2^{s}\right)} .
$$

Proof. Assume that $k \geq 3$ (since the case of $k=2$ is well understood [4]). We will first give a relative simple example for the case of $k=3$ and $s=2$ before proceeding to the general case.

Suppose that for every 2-graph $G$ on $V$, we have

$$
\left|e_{2}(H, G)-e_{2}(\bar{H}, G)\right| \leq \epsilon n^{2} .
$$

We wish to show

$$
\operatorname{dev}_{2} H \leq 32 \epsilon^{1 / 8}
$$


For a vertex $w$, we consider the 2-graph $H_{w}$ with edge set $E\left(H_{w}\right)=\left\{y \in\left(\begin{array}{l}V \\ 2\end{array}\right): y \cup\{w\} \in\right.$ $E(H)\}$. From the definition of $\operatorname{dev}_{2} H$, we have

$$
\operatorname{dev}_{2} H=\frac{1}{n} \sum_{w \in V} \operatorname{dev}_{2} H_{w} .
$$

We consider

$$
S:=\left\{w \in V: \operatorname{dev}_{2} H_{w} \geq 30 \epsilon^{1 / 8}\right\}
$$

If $|S| \leq 2 \epsilon^{1 / 2} n$ then

$$
\operatorname{dev}_{2} H \leq \frac{1}{n}\left(|S|+30 \epsilon^{1 / 8} n\right\} \leq 32 \epsilon^{1 / 8}
$$

as desired. Thus, we may assume $|S| \geq 2 \epsilon^{1 / 2}$.

For each $w \in S$, the fact that $\operatorname{dev}_{2} H_{w} \geq \epsilon^{\prime}=30 \epsilon^{1 / 8}$ implies, by the induction hypothesis using (1) for 2-graphs, that there exists a subset $G_{w}$ (which can be viewed as a 1-graph on $V)$ satisfying

$$
\left|e\left(H_{w}, G_{w}\right)-e\left(\bar{H}_{w}, G_{w}\right)\right|>\delta n^{2}
$$

where $\delta$ satisfies $\delta \geq 16^{-4} \epsilon^{\prime 4} \geq 3 \epsilon^{1 / 2}$. Thus, there is a subset $S^{\prime}$ of $S$ with $\left|S^{\prime}\right|=\epsilon^{1 / 2} n$ so that either

(a) $\left.e\left(H_{w}, G_{w}\right) \geq \frac{1}{2} e\left(\left(\begin{array}{l}V \\ l\end{array}\right)\right), G_{w}\right)+3 \epsilon^{1 / 2} n^{2} / 2$ for all $w \in S^{\prime}$; or

(b) $\left.e\left(H_{w}, G_{w}\right) \leq \frac{1}{2} e\left(\left(\begin{array}{l}V \\ l\end{array}\right)\right), G_{w}\right)-3 \epsilon^{1 / 2} n^{2} / 2$ for all $w \in S^{\prime}$.

We will treat case (a) and omit the similar treatment for case (b).

We proceed to define the following 2-graph $G$ on $V$.

$$
E(G)=\left\{w \cup y: y \in E\left(G_{w}\right)\right\} \backslash\left(\begin{array}{c}
V \backslash S^{\prime} \\
2
\end{array}\right) .
$$

For each $x \in E_{2}(H, G)$, there are three possibilities:

(i) $x$ has at least two vertices in $S^{\prime}$. There are at most $\epsilon n^{3}$ such edges in $E_{2}(H, G)$.

(ii) $x$ has no vertex in $S^{\prime}$. In this case, $x$ can not contain a pair of vertices in $G$, contradicting $x \in E_{2}(H, G)$.

(iii) $x$ has exactly one vertex $w$ in $S^{\prime}$. Say, $x=\{v, u, w\}$ and $u, v \in H_{w}$. Therefore, we have

$$
\begin{aligned}
E_{2}(H, G)-E_{2}\left(\bar{H}_{G}\right) & \geq \sum_{w \in S^{\prime}}\left(E\left(H_{w}, G_{w}\right)-E\left(\bar{H}_{w}, G_{w}\right)\right)-\epsilon n^{3} \\
& \geq\left|S^{\prime}\right| 3 \epsilon^{1 / 2} n^{2} / 2-\epsilon n^{3} \\
& \geq 2 \epsilon n^{2}
\end{aligned}
$$

which is a contradiction. Thus we have proved (2) for the case of $k=3$.

The proof for the general $k$ is quite similar. For a $k$-graph $H$, suppose that for every $(k-1)$-graph $G$ on $V$, we have

$$
\left|e_{s}(H, G)-e_{s}(\bar{H}, G)\right| \leq \epsilon n^{k} .
$$


We wish to show

$$
\operatorname{dev}_{s} H \leq 2^{k+s} \epsilon^{1 /\left((k-s)(k-s+1) 2^{s}\right)} .
$$

For a fixed string of $k-s$ vertices, say, $w=\left(w_{1}, w_{2}, \ldots, w_{k-s}\right)$, we consider edges in $E(H)$ containing $w_{i}$ for $1 \leq i \leq j$. We consider the $(k-i)$-graph $H_{\left(w_{1}, \ldots, w_{i}\right)}$ with edge set $E\left(H_{\left(w_{1}, \ldots, w_{i}\right)}\right)=\left\{y \in\left(\begin{array}{l}V \\ s\end{array}\right): y \cup\left\{w_{1}, \ldots, w_{i}\right\} \in E(H)\right\}$. From the definition of $\operatorname{dev}_{s} H$, we have

$$
\begin{aligned}
\operatorname{dev}_{s} H & =\frac{1}{n} \sum_{w_{1}} \operatorname{dev}_{s} H_{\left(w_{1}\right)} \\
& =\frac{1}{n^{2}} \sum_{w_{1}, w_{2}} \operatorname{dev}_{s} H_{\left(w_{1}, w_{2}\right)} \\
& =\ldots \\
& =\frac{1}{n^{k-s}} \sum_{w=\left(w_{s+1}, \ldots, w_{k}\right)} \operatorname{dev}_{s} H_{w} .
\end{aligned}
$$

For $w_{1} \in V$, we consider

$$
S_{1}:=\left\{w_{1} \in V: \sum_{w_{1}} \operatorname{dev}_{s} H_{w_{1}} \geq\left(2^{k+s}-2\right) \epsilon^{1 /\left((k-s)(k-s+1) 2^{s}\right)}\right\} .
$$

If $|S| \leq 2 \epsilon^{1 /(k-s+1)} n$ then

$$
\operatorname{dev}_{s} H \leq \frac{1}{n}\left(|S|+\left(2^{k+s}-2\right) \epsilon^{1 /\left((k-s)(k-s+1) 2^{s}\right)} n\right\} \leq 2^{k+s} \epsilon^{1 /\left((k-s)(k-s+1) 2^{s}\right)}
$$

as desired. Thus, we may assume $\left|S_{1}\right| \geq 2 \epsilon^{1 /(k-s+1)} n$.

Similarly, it can be shown that for $i=1, \ldots, k-s$, there are subsets $S_{j}$, with $j \leq i$, $\left|S_{j}\right| \geq 2 \epsilon^{1 /(k-s+1)} n$ such that for $\bar{w}_{i}=\left(w_{1}, \ldots, w_{i}\right)$ with $w_{j} \in S_{j}$ for all $j \leq i$, we have $\operatorname{dev}_{s} \bar{H}_{\bar{w}_{i}} \geq\left(2^{k+s}-2^{i}\right) \epsilon^{i /\left((k-s)(k-s+1) 2^{s}\right)}$. In particular, for $w=\left(w_{1}, \ldots, w_{k-s}\right)$ with $w_{i} \in S_{i}$, $1 \leq i \leq k-s$, we have $\operatorname{dev}_{s} H_{w} \geq\left(2^{k+s}-2^{k-s}\right) \epsilon^{1 / 2^{(k-s+1) 2^{s}}}$.

For each $w \in S_{1} \times S_{2} \times \ldots \times S_{k-s}$, the induction hypothesis implies that there exists a $(s-1)$-graph $G_{w}$ on $V$ satisfying

$$
\left|e\left(H_{w}, G_{w}\right)-e\left(\bar{H}_{w}, G_{w}\right)\right|>\delta n^{s}
$$

where $\delta$ satisfies $\delta=4^{-s}\left(2^{k+s-1}\right)^{2^{s}} \epsilon^{1 /(k-s+1)}$.

Thus, there are subsets $S_{i}^{\prime}$ of $S_{i}, 1 \leq i \leq k-s$, with $\left|S_{i}^{\prime}\right|=\epsilon^{1 /(k-s+1)} n$ so that for $w \in S^{\prime}=S_{1}^{\prime} \times S_{2}^{\prime} \times \ldots \times S_{k-s}^{\prime}$ either

(a) $e\left(H_{w}, G_{w}\right)-e\left(\bar{H}_{w}, G_{w}\right) \geq \delta n^{s}$ for all $w \in S^{\prime}$; or

(b) $e\left(H_{w}, G_{w}\right)-e\left(\bar{H}_{w}, G_{w}\right) \leq-\delta n^{s}$ for all $w \in S^{\prime}$.

We will treat case (a) and omit the similar treatment for case (b).

We proceed to define the following $(k-1)$-graph $G$ on $V$.

$$
E(G)=\left\{w \cup y: y \in E\left(G_{w}\right)\right\} \backslash\left(\begin{array}{c}
V \backslash\left(S_{1}^{\prime} \cup \ldots \cup S_{k-s}^{\prime}\right) \\
s-1
\end{array}\right) .
$$

Random Structures and Algorithms DOI 10.1002/rsa 
For each $x \in E_{s}(H, G)$, there are three possibilities:

(i) $x$ contains more than one vertex in some $S_{i}^{\prime}$. There are at most $\epsilon n^{k}$ such edges.

(ii) $x$ has no vertex in $S_{i}^{\prime}$ for some $i$. In this case, $x$ can not contain any edge in $G$, contradicting $x \in E_{s}(H, G)$.

(iii) $x$ has exactly one vertex $w_{i}$ in $S_{i}^{\prime}$ for $i=1, \ldots, k-s$. Say, $x=w \cup x^{\prime}$, where for any vertex $u \in x^{\prime}$ we have $x\{u\} \in E(G)$. Therefore, we have

$$
\begin{aligned}
E_{s}(H, G)-E_{s}\left(\bar{H}_{G}\right) & \geq \sum_{w \in S^{\prime}}\left(E\left(H_{w}, G_{w}\right)-E\left(\bar{H}_{w}, G_{w}\right)\right)-\epsilon n^{k} \\
& \geq \prod_{i=1}^{k-s}\left|S_{i}^{\prime}\right| \cdot \delta n^{s}-\epsilon n^{k} \\
& \geq \epsilon^{(k-s) /(k-s+1)} \cdot 3 \epsilon^{1 /(k-s+1)} n^{k}-\epsilon n^{k} \geq \epsilon n^{k}
\end{aligned}
$$

which is a contradiction. This completes the proof for (2).

Combining the above two theorem, we see that $\operatorname{dev}_{s}$ and $\operatorname{disc}^{(s)}$ are equivalent.

\section{CONCLUDING REMARKS}

In a $k$-graph $H$, many questions can be asked concerning the $(l, s)$-discrepancy properties $\operatorname{disc}_{l}^{(s)}$. For example, we have, for $s \geq 3$,

$$
\operatorname{disc}_{k}^{(s)} \Rightarrow \operatorname{disc}_{k}^{(s-1)}
$$

by using the fact that $\operatorname{dev}_{l} \Rightarrow \operatorname{dev}_{l-1}$ and the main theorem $\operatorname{disc}_{k}^{(s)} \Leftrightarrow \operatorname{dev}_{s}$. However, in a $k$-graph and $2 \leq l<k$, is it true that

$$
\operatorname{disc}_{l}^{(s)} \Rightarrow \operatorname{disc}_{l}^{(s-1)} ?
$$

In the implication (3), the reversed direction does not hold (see [2]). For a general $l$ with $l<k$, is it still true? Is it possible to have one equivalence class which includes $\operatorname{disc}_{l}^{(s)}$ for some consecutive values of $s$ for some $l$ ? What is then the length of the chain of equivalence classes containing $\operatorname{disc}_{l}^{(s)}$ as $s$ ranges from 1 to $\left(\begin{array}{c}k \\ l-1\end{array}\right)$ ?

Recall that $\operatorname{disc}_{l}=\operatorname{disc}_{l}^{(s)}$ with $s=\left(\begin{array}{c}k \\ l-1\end{array}\right)$. From the definition, it is not hard to check that $\operatorname{disc}_{l} \Rightarrow \operatorname{disc}_{l-1}$ for $l \geq 3$. To further explore the relations among $\operatorname{disc}_{l}^{(s)}$ and $\operatorname{disc}_{l-1}^{(t)}$, we need more definitions.

We consider a $k$-graph $H$ with vertex set $V=V(H)$ and $E=E(H)$. Let $Q$ denote a fixed $l$-graph on $k$ vertices and $G$ denote a $l$-graph on vertex set $V$. In a $k$-graph $H$, an edges $x$ in $E(H)$ is said to be $Q$-induced by $G$ if there is an embedding $\pi$ of $Q$ into $\left(\begin{array}{l}x \\ l\end{array}\right)$, the set of $l$-subsets of $x$ satisfying the property that for all $y \in E(Q)$, the images $\pi(y)$ are in $E(G)$. Let $e_{Q}(H, G)$ denote the total number of edges in $H$ which are $Q$-induced by $G$.

Definition. For a $k$-graph $H$ on vertex set $V$ with $|V|=n$ and a fixed $(l-1)$-graph $Q$, we define $\operatorname{disc}_{l}^{Q} H$ by:

$$
\operatorname{disc}_{l}^{Q} H=\frac{1}{n^{k}} \max _{M}\left|e_{Q}(H, G)-e_{Q}(\bar{H}, G)\right|,
$$

where the maximum is taken over all $(l-1)$-graphs $G$ on $V$. 
It is of interest to examine possible necessary and sufficient conditions for a pair of graphs $Q$ and $Q^{\prime}$ on $k$ vertices such that $\operatorname{disc}_{l}^{Q} \Leftrightarrow \operatorname{disc}_{l}^{Q^{\prime}}$. For a fixed $k$ and a graph $Q$, how large is the family of graphs consisting of $Q^{\prime}$ satisfying the property that $\operatorname{disc}_{l}^{Q^{\prime}}$ is in the quasi-random class that includes $\operatorname{disc}_{l}^{Q}$ ? How are properties $\operatorname{disc}_{l}^{Q}$ related to $\operatorname{disc}_{l}^{(s)}$ ? Most of all, what is the lattice structure illustrating the relations among quasi-random classes of $k$-graphs? In this note, we only example some very special parts of this lattice. Numerous questions remain to be explored.

\section{REFERENCES}

[1] F. R. K. Chung, Quasi-random classes of hypergraphs, Random Struct Algorithms 1 (1990), 363-382.

[2] F. R. K. Chung and R. L. Graham, Cohomological aspects of hypergraphs, Trans Am Math Soc 334 (1992), 365-388.

[3] F. R. K. Chung and R. L. Graham, Quasi-random set systems, J Am Math Soc 4 (1991), 151-196.

[4] F. R. K. Chung, R. L. Graham, and R. M. Wilson, Quasi-random graphs, Combinatorica 9 (1989), 345-362.

[5] F. R. K. Chung and P. Tetali, Communication complexity and quasi randomness, SIAM J Discrete Math 6 (1993), 110-123.

[6] D. Conlon, H. Han, Y. Person, and M. Schacht, Weak quasi-randomness for uniform hypergraphs, Random Struct Algorithms 40 (2012), 1-38.

[7] Y. Kohayakawa, V. Rödl, and J. Skokan, Hypergraphs, quasi-randomness, and conditions for regularity, J Combinatorial Theory Ser A 97 (2002), 307-352.

[8] V. Rödl and J. Skokan, Counting subgraphs in quasi-random 4-uniform hypergraphs, Random Struct Algorithms 26 (2005), 160-203. 